\title{
Quantitative Analysis of the Cervical Texture by Ultrasound and Correlation with
}

\section{Gestational Age}

Núria Baños ${ }^{1}$, Alvaro Perez-Moreno ${ }^{2}$, Federico Migliorelli ${ }^{1}$, Laura Triginer ${ }^{2}$, Teresa Cobo $^{1,3}$, Elisenda Bonet-Carne ${ }^{2}$, Eduard Gratacos ${ }^{1,3}$, Montse Palacio ${ }^{1,3}$.

${ }^{1}$ BCNatal - Barcelona Center for Maternal-Fetal and Neonatal Medicine (Hospital Clínic and Hospital Sant Joan de Deu), Fetal i+D Fetal Medicine Research Center, IDIBAPS, University of Barcelona, Spain.

2 Transmural Biotech S. L. Barcelona, Spain.

${ }^{3}$ Center for Biomedical Research on Rare Diseases (CIBER-ER), Barcelona, Spain.

Short title: Cervical Texture and correlation with gestational age.

Key words: Quantitative ultrasound, Uterine cervix, Image biomarker.

\section{Corresponding author:}

Núria BAÑOS, MD

BCNatal | Barcelona Center for Maternal Fetal and Neonatal Medicine

Hospital Clínic and Hospital Sant Joan de Déu, Universitat de Barcelona

Fetal i+D Fetal Medicine Research Center, IDIBAPS

Sabino de Arana 1, 08028 Barcelona, Spain

Work Phone. +34 932275400 EXT. 7353

Cell Phone: +34 699159706

Mail:nbanos@clinic.cat 


\begin{abstract}
Objectives

Quantitative texture analysis has been proposed to extract robust features from the ultrasound image to detect subtle changes in the textures of the images. The aim of this study was to evaluate the feasibility of quantitative cervical textures analysis to quantify cervical tissue changes throughout pregnancy.
\end{abstract}

\title{
Methods
}

This was a cross-sectional study including singleton pregnancies between 20.0 and 41.6 weeks of gestation from women who delivered at term. Cervical length was measured and a selected region of interest (ROI) in the cervix was delineated. A model to predict gestational age based on features extracted from cervical images was developed following three steps: data splitting, feature transformation and regression model computation.

\section{Results}

700 images, 30 per gestational week, were included for analysis. There was a strong correlation between the gestational age at which the images were obtained and the estimated gestational age by quantitative analysis of the cervical texture $(\mathrm{R}=0.88)$.

\section{Discussion}

This study provides evidence that quantitative analysis of cervical texture can extract features from cervical ultrasound images which are correlated to gestational age. Further research is needed to evaluate its applicability as a biomarker of the risk of SPTB, as well as its role in cervical assessment in other clinical situations in which cervical evaluation might be relevant. 


\section{Introduction}

Cervical ripening is a chronic process, consisting in microstructural and water concentration changes, which starts during the first trimester and progressively proceeds until term in a normal pregnancy. Four differentiated phases have been described: softening, ripening, dilation and repair. Initially there is a progressive softening of the cervix beginning early in pregnancy, due to a reorganization of the collagen fibrillar network. There is also an increased in the vascularity and oedema of the entire cervix, a hypertrophy of cervical stroma and hypertrophy and hyperplasia of the cervical glands. Shortening and dilatation occur as later events, several days before delivery [1] [2]. Nonetheless, these major alterations in the extracellular matrix and cellular composition of cervical tissue taking place along pregnancy are subtle and difficult to detect by a clinical or routine sonographic examination.

Appropriate timing in cervical function and composition during gestation are crucial for a successful pregnancy. Premature cervical remodelling and ripening usually precede a spontaneous preterm birth regardless of the initial etiology [3], and an unripe cervix will probably lead to a failed induction of labor (IOL) [4]. With the lack of good tools to assess cervical remodeling [5], there is growing scientific interest to learn more about cervical structure and behaviour.

Several studies have suggested that women with a short cervix $(\leq 20 \mathrm{~mm})$ in the midtrimester of pregnancy are at increased risk for spontaneous preterm birth [6]. Nevertheless, the prevalence of short cervical length and its sensibility for the detection of SPTB are extremely low [7-9]. Consequently, only a small proportion of pregnant women are identified as being at high risk of preterm delivery and potentially benefit from the treatments available [10-12]. For these reasons, the value of cervical length as a universal screening tool remains controversial. Therefore, other objective methods or 
strategies aimed at detecting early stages of cervical remodelling in order to better identify women at high risk are needed.

Quantitative texture analysis by ultrasound has demonstrated to extract robust features related to subtle differences in the textures of ultrasound images. Texture analysis by ultrasound or magnetic resonance (MR) has also been investigated in the field of foetal and perinatal medicine $[13,14]$. Recently, quantitative texture analysis of foetal lung ultrasound images has proven to be a predictor of neonatal respiratory morbidity [15-17]. The aim of this study was to evaluate the feasibility of quantitative analysis of cervical ultrasound images to evaluate cervical tissue changes throughout pregnancy.

\section{Methods}

This was a cross-sectional study including singleton pregnancies between 20.0 and 41.6 weeks of gestation attending the Barcelona Center for Maternal Fetal and Neonatal Medicine in Hospital Clínic and Hospital Sant Joan de Déu in Barcelona for routine pregnancy ultrasound scans from March 2014 to January 2015. Multiple pregnancies, patients who received any treatment to prevent SPTB (progesterone or cervical cerclage) and women who delivered $<37$ weeks were excluded from the study. Demographic characteristics, obstetrical history, gestational age at delivery and mode of delivery were collected. The study protocol was approved by the local Ethics Committee (ID HCB 2014/0089), and all pregnant women provided written informed consent. Gestational age was calculated based on the crown-rump length at first trimester ultrasound.

\section{Image Acquisition and Region of Interest}


An image acquisition guide was designed in order to ensure the optimal acquisition parameters for further analysis of the images: A sagittal view of the cervix without exerting any pressure with the transducer, so that the diameter of the anterior and posterior cervical lips were similar, was obtained. The internal and external os, as well as the cervical canal should be identified and the entire cervical structure visualized, avoiding zoom and using only depth function. The cervix should be located horizontally to the transducer. Calipers, shadows and saturations must also be avoided. Images had to be obtained without any post-processing function, such as speckle reduction imaging (SRI), smoothing and on-line measurements as shown in figure 1a. Gain and harmonics were at the discretion of the physician. Siemens Sonoline Antares (Siemens Medical Systems, Malvern, Pa., USA), Voluson 780 Pro, Voluson S6, Voluson E6 and Voluson E8 (GE Medical Systems, Milwaukee, Wisc., USA) with a vaginal probe with a frequency between 2-10 MHz were used to acquire the images. Data were digitally collected in the original Digital Imaging and Communication in Medicine (DICOM) format and stored for off-line analysis in a custom made program with a Graphic User Interface (GUI) using MATLAB R2010b (version 7.11.0.584; MATLAB; The Mathworks Inc., Natick, Mass., USA). Images were acquired by experienced sonographers. The acquired images underwent a quality control process before delineation, according to the acquisition guide criteria. Cervical length was measured from the outer to the inner cervical os following the established guidelines [18]. The region of interest (ROI) was defined has the largest homogeneous region of the middle part of the anterior cervical lip. ROI delineation should avoid the cervical canal, the glandular area and the internal and external cervical os (figure 1b). Cervical length measurement and manual free hand delineation of the ROI were performed by two independent operators (NB, AP). Subsequently, the delineations 
were reviewed by a third operator (LT) in order to confirm that they met the delineation criteria.

\section{Feature extraction}

Quantitative texture analysis was performed to extract features from the delineated ROI. Feature extraction was performed by means of a Low Binary Patterns (LBP) algorithm [19], concretely a multiresolution gray-scale and rotation invariant approach mainly based on recognizing that local binary patterns are local image texture properties. A total of 18 cervical ultrasound features were obtained from each ultrasound image and ROI.

\section{Statistical analysis and learning procedure}

Once features were extracted by quantitative texture analysis from cervical ultrasound images, a model of gestational age was developed following next three steps: data splitting, feature transformation, and regression model computation. Regarding the statistical learning algorithm, the data was split into two bags. A cohort of women with gestational age at acquisition below 23 and above 39 weeks of gestation was included in Bag1a and Bag1b, respectively. The cohort of women between 23.0 and 38.6 weeks of gestational age was included in Bag2.

With respect to the feature transformation algorithm, feature selection and dimension reduction were performed from all Bag1 images in order to provide the most relevant features related to gestational age since they represent both ends in the cervical remodelling process. The feature selection was performed by means of the ratio of mean to standard deviation between extracted features. Once features were selected, the dimension reduction step was performed by means of principal component analysis. 
Finally, a regression model was computed using Bag2 images to test the robustness and relevance of the transformed features extracted by the LBP algorithm from the Bag1 to quantify cervical texture changes. The random forest was the learning method used to train a regression model for estimating weeks of gestation. We ensured that the trained model was not over-fitted since the number of features used for training was less than one third of the sample size [20].

The model of gestational age based on features extracted by quantitative texture analysis was evaluated using regression fitting indicators as the Pearson correlation $\left(\mathrm{R}^{2}\right)$ and root mean square error (RMSE). Lin's Concordance Correlation coefficient and BlandAltman plot were also used to test the agreement between the real and the estimated gestational age by texture analysis. Demographic characteristics were described as absolute and relatives frequencies for qualitative variables and mean and standard deviation for quantitative variables. Additional clinical statistical analyses were computed with STATA/IC 13.0 (StataCorp 4905 Lakeway Drive College Station, Texas, USA). Statistical learning analyses were performed using MATLAB R2010b (version 7.11.0.584; MATLAB; The Mathworks Inc., Natick, Mass., USA).

\section{Results}

From March 2014 to January 2015 a total of 903 images between 20.0 and 41.6 weeks of pregnancy were acquired. After reviewing the perinatal outcomes 49 women who delivered preterm were excluded, and 154 images were excluded after the image acquisition quality control. The main reason for exclusion was the presentation of a non-horizontal cervical canal. After the ROI delineation, all images met the required criteria and no re-delineations were necessary. Thus, 700 images with approximately 30 
images per gestational week were finally included for analysis. The demographic characteristics and pregnancy outcome of the study population are summarized in table 1.

The feature transformation algorithm obtained was first trained with the features from 189 images, 84 images below 23 weeks of gestation (bag 1a) and 105 images above 39 weeks of gestation (bag 1b). A regression model to correlate the features obtained with the gestational age was then evaluated in a large cohort of 511 images between 23.0 and 38.6 weeks of gestational age. The sample distribution by weeks of gestation is shown in figure 2. There was a strong correlation between the real gestational age and the estimated gestational age by texture analysis $(\mathrm{R}=0.88)$ as shown in figure 3. Lin's Concordance Correlation coefficient was 0.87 , showing a high absolute agreement. Bland-Altman interval of agreement was -0.13 (95\% CI -4.52 to 4.26$)$, with $3.52 \%$ of the cases over the limit and $1.37 \%$ of the cases under the limit (figure 4).

Cervical length was measured in the same cohort of images in which quantitative analysis of cervical ultrasound texture and regression model computation were performed (Bag 2). The correlation between gestational age and cervical length measurements was $R=-$ 0.26. (figure 5). Table 2 shows the correlation of the real gestational age with both, the estimated gestational age by quantitative analysis of cervical texture and cervical length measurement.

\section{Discussion}

This study provides evidence that quantitative analysis of cervical texture can feasibly extract features from cervical ultrasound images related to gestational age. 
Pearson correlation coefficient (0.88) and Bland-Altman plot showed a good agreement between the real gestational age and the estimated gestational age by texture analysis. Considering that the cervical microstructure progressively changes throughout pregnancy until the time of delivery, gestational age could be assessed as a surrogate of these cervical changes. Assuming certain degree of cervical structure variability within women in the same gestational age, the observed dispersion when comparing both approaches could be explained by this inherent biological variability. Therefore, the feasibility of quantifying cervical changes from the 20.0 to 41.6 weeks of pregnancy represents a breakthrough in the detection of cervical remodelling by ultrasound.

The changes in quantitative analysis of cervical textures were also compared to the changes in cervical length throughout pregnancy in the same group of images. Cervical texture correlates with gestational age much better than cervical length ( $\mathrm{R}$ cervical length $=-0.26$ vs. $\mathrm{R}$ Cervical texture $=0.88$ ). These results suggest that quantitative analysis of cervical texture is capable of detecting cervical changes along pregnancy while cervical length is not, as it remains virtually stable. In line with our findings, several studies have reported few changes in cervical length throughout pregnancy, especially between 17 and 32 gestational weeks [21] with a high variability and dispersion between studies at the end of the pregnancy [22] [23]. Indeed, most of these studies showed a weak negative correlation between cervical length and gestational age [24,25], which supports its limited usefulness in detecting early cervical remodelling.

A strength of the present study is the large number of images included, with a homogeneous distribution all along pregnancy. The demographic characteristics of all the patients as well as their perinatal outcomes were recorded in order to exclude patients who delivered preterm from the final sample. This guarantees that, from the clinical point 
of view, features were extracted from cervical images representing the expected cervical remodelling along a normal pregnancy.

This study presents some limitations. Firstly, the robustness in terms of using different scanner equipment and frequency probe was assumed. Although the robustness of these feature extraction and statistical learning procedure has already been proven in previous studies[16], further research with this specific setting is needed. Secondly, the ROI used in this study was selected because it is an easy delineation, as the anterior lip is always identifiable. Moreover, it avoids shadows and alterations produced by the cervical canal and mucus, since this region is the part of the cervix closest to the transducer. However, other ROI should be analyzed in order to evaluate the whole cervical structure with this same quantitative method and determine the most appropriate ROI.

In recent years there has been a growing interest concerning the evaluation of uterine cervix properties in order to better understand its behaviour and role throughout pregnancy. To date, elastography has been the method most studied, with several publications assessing its measurement standardization, reproducibility and performance in the prediction of preterm birth risk and successful IOL. Elastography measures the percentage of tissue deformation occurring when oscillatory compression is applied, and this deformation can be expressed as strain [26,27]. However, strain measurements and colour elastograms are related to the displacement of structures, which are directly related to the force exterted by the operator. Thus, a more objective method, independent of the pressure made by operator is necessary in order to obtain more reproducible results.

The Cervical Consistency Index (CCI) assesses another biomechanical property of the uterine cervix, the maximum tissue deformability after manual compression of the cervix with the vaginal probe. Pressure is applied softly and progressively on the cervix until no 
further shortening of the anteroposterior diameter is observed. The CCI is a reproducible measurement, although the force applied to the tissue is also unknown[28]. The quantitative measure is calculated as the ratio between the anteroposterior diameter at maximum compression and the diameter at rest [29]. The CCI has shown a clear inverse linear relationship with gestational age. The CCI has also been found to be lower in women who delivered preterm and appeared to provide a better prediction of spontaneous preterm birth than does cervical length [28]. Nonetheless, these findings need to be validated in a larger external cohort.

Other methods are able to assess cervical biomechanical characteristics such as distensibility and tissue stiffness [30-36], uterine cervix composition and optical characteristics [37-43]. Unfortunately, most of these methods are invasive and evaluate only a specific part of the cervix, which is an important limitation taking into account the heterogeneity of the uterine cervix structure.

The methods developed to date have demonstrated histological, biochemical or biomechanical changes in the uterine cervix, but none have met the requirements to be implemented in clinical practice as predictive tools.

It is important to highlight that quantitative analysis of cervical texture by ultrasound overcomes most of these technical limitations, as it is an objective non-invasive method, which does not require special equipment and training and could potentially evaluate the whole uterine cervix.

In conclusion, this study lays solid groundwork to explore quantitative analysis of cervical texture in clinical scenarios to evaluate cervical ripening and identifying changes undetectable by cervical length or clinical examination. The feasibility of the quantitative analysis of cervical texture to detect changes throughout pregnancy facilitates its potential 
implementation to predict clinical outcomes such as spontaneous preterm birth or failed IOL. Nonetheless, further research is needed to evaluate its ability as an image biomarker. 


\section{Acknowledgements}

This publication has been funded with support of the Erasmus + Programme of the European Union (Framework Agreement number: 2013-0040) [This publication reflects the views only of the author, and the Commission cannot be held responsible for any use which may be made of the information contained therein]. Additionally, the research leading to these results has partially received funding from "la Caixa" Foundation; Cerebra Foundation for the Brain Injured Child (Carmarthen, Wales, UK); AGAUR 2014 SGR grant n 928 and "la Secretaria d'Universitats i Recerca del Departament d'Economia i Coneixement de la Generalitat de Catalunya (A.P: 2014DI083)". 


\section{Refererences}

$1 \quad$ Word RA, Li XH, Hnat M, Carrick K: Dynamics of cervical remodeling during pregnancy and parturition: Mechanisms and current concepts. Semin Reprod Med 2007;25:69-79.

2 Timmons $B$, Akins $M$, Mahendroo $M$ : Cervical remodeling during pregnancy and parturition. Trends Endocrinol Metab 2010;21:353-361.

3 Romero R, Dey SK, Fisher SJ: Preterm labor: One syndrome, many causes. Science 2014;345:760-765.

4 Banos N, Migliorelli F, Posadas E, Ferreri J, Palacio M: Definition of failed induction of labor and its predictive factors: Two unsolved issues of an everyday clinical situation. Fetal Diagn Ther 2015;38:161-169.

5 Berghella V, Baxter JK, Hendrix NW: Cervical assessment by ultrasound for preventing preterm delivery. Cochrane Database Syst Rev 2013;1:CD007235.

6 Fonseca EB, Celik E, Parra M, Singh M, Nicolaides KH: Progesterone and the risk of preterm birth among women with a short cervix. N Engl J Med 2007;357:462-469.

7 Kuusela P, Jacobsson B, Soderlund M, Bejlum C, Almstrom E, Ladfors L, Hagberg H, Wennerholm UB: Transvaginal sonographic evaluation of cervical length in the second trimester of asymptomatic singleton pregnancies, and the risk of preterm delivery. Acta Obstet Gynecol Scand 2015;94:598-607.

8 Orzechowski KM, Boelig RC, Baxter JK, Berghella V: A universal transvaginal cervical length screening program for preterm birth prevention. Obstet Gynecol 2014;124:520-525.

9 van der Ven J, van Os MA, Kazemier BM, Kleinrouweler E, Verhoeven CJ, de Miranda E, van Wassenaer-Leemhuis AG, Kuiper PN, Porath M, Willekes C, Woiski MD, Sikkema MJ, Roumen FJ, Bossuyt PM, Haak MC, de Groot CJ, Mol BW, Pajkrt E: The capacity of midpregnancy cervical length to predict preterm birth in low-risk women: A national cohort study. Acta Obstet Gynecol Scand 2015;94:1223-1234.

10 Romero R, Yeo L, Chaemsaithong P, Chaiworapongsa T, Hassan SS: Progesterone to prevent spontaneous preterm birth. Semin Fetal Neonatal Med 2014;19:15-26.

11 Celik E, To M, Gajewska K, Smith GC, Nicolaides KH: Cervical length and obstetric history predict spontaneous preterm birth: Development and validation of a model to provide individualized risk assessment. Ultrasound Obstet Gynecol 2008;31:549-554.

12 Berghella V, Rafael TJ, Szychowski JM, Rust OA, Owen J: Cerclage for short cervix on ultrasonography in women with singleton gestations and previous preterm birth: A metaanalysis. Obstet Gynecol 2011;117:663-671.

13 Sanz-Cortes M, Ratta GA, Figueras F, Bonet-Carne E, Padilla N, Arranz A, Bargallo N, Gratacos E: Automatic quantitative mri texture analysis in small-for-gestational-age fetuses discriminates abnormal neonatal neurobehavior. PLoS One 2013;8:e69595.

14 Tenorio V, Bonet-Carne E, Botet F, Marques F, Amat-Roldan I, Gratacos E: Correlation between a semiautomated method based on ultrasound texture analysis and standard ultrasound diagnosis using white matter damage in preterm neonates as a model. J Ultrasound Med 2011;30:1365-1377.

15 Cobo T, Bonet-Carne E, Martinez-Terron M, Perez-Moreno A, Elias N, Luque J, AmatRoldan I, Palacio M: Feasibility and reproducibility of fetal lung texture analysis by automatic quantitative ultrasound analysis and correlation with gestational age. Fetal Diagn Ther 2012;31:230-236.

16 Bonet-Carne E, Palacio M, Cobo T, Perez-Moreno A, Lopez M, Piraquive JP, Ramirez JC, Marques F, Gratacos E: Quantitative ultrasound texture analysis of fetal lungs to predict neonatal respiratory morbidity. Ultrasound Obstet Gynecol 2015 
17 Palacio M, Cobo T, Martinez-Terron M, Ratta GA, Bonet-Carne E, Amat-Roldan I, Gratacos E: Performance of an automatic quantitative ultrasound analysis of the fetal lung to predict fetal lung maturity. Am J Obstet Gynecol 2012;207:504 e501-505.

18 Kagan KO, Sonek J: How to measure cervical length. Ultrasound Obstet Gynecol 2015;45:358-362.

19 Timo Ojala MPaTMI: Multiresolution gray-scale and rotation invariant texture classification with local binary patterns. Transactions on Pattern Analysis and Machine Intelligence, 2002; 24:971-987.

20 Hastie T TR, J Friedman:: The elements of statistical learning: Data mining, inference, and prediction. ed 2 New York, Springer 2009

21 Rovas L, Sladkevicius $P$, Strobel E, Valentin L: Reference data representative of normal findings at two-dimensional and three-dimensional gray-scale ultrasound examination of the cervix from 17 to 41 weeks' gestation. Ultrasound Obstet Gynecol 2006;27:392-402.

22 Gramellini D, Fieni S, Molina E, Berretta R, Vadora E: Transvaginal sonographic cervical length changes during normal pregnancy. J Ultrasound Med 2002;21:227-232; quiz 234-225.

23 Brieger GM, Ning XH, Dawkins RR, Ying KQ, Weng C, Chang AM, Haines CJ: Transvaginal sonographic assessment of cervical dynamics during the third trimester of normal pregnancy. Acta Obstet Gynecol Scand 1997;76:118-122.

24 Hoesli IM, Strutas D, Tercanli S, Holzgreve W: Charts for cervical length in singleton pregnancy. Int J Gynaecol Obstet 2003;82:161-165.

25 Tongsong $\mathrm{T}$, Kamprapanth $\mathrm{P}$, Pitaksakorn J: Cervical length in normal pregnancy as measured by transvaginal sonography. Int J Gynaecol Obstet 1997;58:313-315.

26 Hernandez-Andrade E, Hassan SS, Ahn H, Korzeniewski SJ, Yeo L, Chaiworapongsa T, Romero R: Evaluation of cervical stiffness during pregnancy using semiquantitative ultrasound elastography. Ultrasound Obstet Gynecol 2013;41:152-161.

27 Hernandez-Andrade E, Romero R, Korzeniewski SJ, Ahn H, Aurioles-Garibay A, Garcia $M$, Schwartz AG, Yeo L, Chaiworapongsa T, Hassan SS: Cervical strain determined by ultrasound elastography and its association with spontaneous preterm delivery. J Perinat Med 2014;42:159-169.

28 Parra-Saavedra M, Gomez L, Barrero A, Parra G, Vergara F, Navarro E: Prediction of preterm birth using the cervical consistency index. Ultrasound Obstet Gynecol 2011;38:44-51.

29 Mazza E, Parra-Saavedra M, Bajka M, Gratacos E, Nicolaides K, Deprest J: In vivo assessment of the biomechanical properties of the uterine cervix in pregnancy. Prenat Diagn 2014;34:33-41.

30 Cabrol D, Carbonne B, Jannet D, Baton C, Bonoris E, Dudzik W, Lehouezec R: Prognostic value of cervical distensibility index measurement in the outcome of pregnancies with threatened premature labor. Gynecol Obstet Invest 1991;32:28-32.

31 Cabrol D, Carbonne B, Ledieu C, Lussiana F: Relationship between mechanical properties of the uterine cervix and occurrence of postdate pregnancy. Gynecol Obstet Invest 1991;32:36-38.

32 Anthony GS, Walker RG, Robins JB, Cameron AD, Calder AA: Management of cervical weakness based on the measurement of cervical resistance index. Eur J Obstet Gynecol Reprod Biol 2007;134:174-178.

33 Hee L, Liao D, Sandager P, Gregersen H, Uldbjerg N: Cervical stiffness evaluated in vivo by endoflip in pregnant women. PLoS One 2014;9:e91121.

34 Bauer M, Mazza E, Jabareen M, Sultan L, Bajka M, Lang U, Zimmermann R, Holzapfel GA: Assessment of the in vivo biomechanical properties of the human uterine cervix in pregnancy using the aspiration test: A feasibility study. Eur J Obstet Gynecol Reprod Biol 2009;144 Suppl 1:S77-81.

35 Bauer M, Mazza E, Nava A, Zeck W, Eder M, Bajka M, Cacho F, Lang U, Holzapfel GA: In vivo characterization of the mechanics of human uterine cervices. Ann N Y Acad Sci 2007;1101:186-202. 
36 Tekesin I, Hellmeyer L, Heller G, Romer A, Kuhnert M, Schmidt S: Evaluation of quantitative ultrasound tissue characterization of the cervix and cervical length in the prediction of premature delivery for patients with spontaneous preterm labor. Am J Obstet Gynecol 2003;189:532-539.

37 O'Connell MP, Avis NJ, Brown BH, Killick SR, Lindow SW: Electrical impedance measurements: An objective measure of prelabor cervical change. J Matern Fetal Neonatal Med 2003;14:389-391.

38 Banos A, Wolf M, Grawe C, Stahel M, Haensse D, Fink D, Hornung R: Frequency domain near-infrared spectroscopy of the uterine cervix during cervical ripening. Lasers Surg Med 2007;39:641-646.

39 Maul H, Olson G, Fittkow CT, Saade GR, Garfield RE: Cervical light-induced fluorescence in humans decreases throughout gestation and before delivery: Preliminary observations. Am J Obstet Gynecol 2003;188:537-541.

40 Fittkow CT, Maul H, Olson G, Martin E, Mackay LB, Saade GR, Garfield RE: Lightinduced fluorescence of the human cervix decreases after prostaglandin application for induction of labor at term. Eur J Obstet Gynecol Reprod Biol 2005;123:62-66.

41 McFarlin BL, Bigelow TA, Laybed Y, O'Brien WD, Oelze ML, Abramowicz JS: Ultrasonic attenuation estimation of the pregnant cervix: A preliminary report. Ultrasound Obstet Gynecol 2010;36:218-225.

42 Kuwata T, Matsubara S, Taniguchi N, Ohkuchi A, Ohkusa T, Suzuki M: A novel method for evaluating uterine cervical consistency using vaginal ultrasound gray-level histogram. J Perinat Med 2010;38:491-494.

43 Afzali N, Mohajeri M, Malek A, Alamatian A: Cervical gland area: A new sonographic marker in predicting preterm delivery. Arch Gynecol Obstet 2012;285:255-258. 


\section{FIGURES:}

Figure 1. Sagittal view of the cervix (a) and delineation of the anterior cervical lip (b). Figure 2. Histogram and distribution of the final sample size.

Figure 3. Relationship between the estimated gestational age by quantitative analysis of cervical textures (weeks) and the real gestational age (weeks). R Pearson correlation was 0.88 .

Figure 4. Bland-Altman plot showing the agreement between the estimated gestational by texture analysis and the real gestational age

Figure 5. Relationship between the cervical length $(\mathrm{mm})$ and the real gestational age (weeks). R Pearson correlation was -0.26 . 